\title{
Reclamaciones españolas: índice de expedientes fallados*
}

Carlos Illades

INSTITUTO MORA

$\mathbf{L}$

a lucha armada iniciada en 1910 causó daños de consideración a las propiedades de mexicanos y extranjeros quienes, con insistencia, se quejaron ante las autoridades gubernamentales y recurrieron a distintas formas de presión para que los daños fueran reparados. Con este fin, los gobiernos de Francisco León de la Barra, primero, y de Venustiano $\mathrm{Ca}$ rranza, después, se comprometieron a crear instancias ad boc para atender las reclamaciones de los daños causados por la revolución.

Los inmigrantes españoles, una de las minorías extranjeras más afecta-

* Este trabajo fue financiado por el ministerio de Trabajo de España y forma parte del proyecto "España en América a través de sus inmigrantes", coordinado por el Dr. Álvaro Matute. das por los acontecimientos armados, presentaron ante la Comisión Mixta Hispano-Mexicana de Reclamaciones (CMHMR), formada en 1925, más de mil reclamaciones por daños a propiedades y personas. Un número apreciable de éstas forma parte del acervo del Archivo Histórico de la Embajada de España en México, (AHEEM), microfilmado por la Biblioteca Daniel Cosío Villegas de El Colegio de México.

A continuación se presenta un índice de los expedientes fallados por la Comisión Mixta Hispano-Mexicana de Reclamaciones. Para facilitar su consulta, se numeraron consecutivamente éstos y se anexaron los datos de la entidad en la cual se dice ocurrió el daño, así como el año en que se integró el expediente de la reclamación. Cabe aclarar que, cuando un expediente 
comienza en un r. y termina en otro, se consigna solamente en el primero.

\section{COMISIÓN MIXTA HISPANO-MEXICANA B. ARCHIVO DE EXPEDIENTES}

1. "España en nombre de Juan Pría Álvarez contra los Estados Unidos Mexicanos."

Año: s.f.

Estado: Distrito Federal

Archivo Histórico de la Embajada de España en México (AHEEM), Comisión Mixta Hispano-Mexicana de Reclamaciones (СМHMR): MP 142, r. 135, c. 1.

2. "España en nombre de Andrés Fernández de la Maza contra los Estados Unidos Mexicanos."

Año: s.f.

Estado: Veracruz

AHEEM, CMHMR: MP 142, r. 135, c. 1.

3. "España en nombre de Dámaso Crespo Arteaga contra los Estados Unidos Mexicanos."

Año: 1932

Estado: Tabasco

AHEEM, CMHMR: MP 142, r. 136, c. 1.

4. "España en nombre de Anastasio y herederos de Sabino Briz como socios de A. Briz contra los Estados Unidos Mexicanos."

Año: 1931

Estado: Chiapas

AHEEM, CMHMR: MP 142, r. 136, c. 1.

5. "España en nombre de Francisco Armendártz sucesores contra los Estaclos Unidos Mexicanos."

Año: 1932
Estado: Tamaulipas

AHEEM, CMHMR: MP 142, r. 136, c. 2.

6. "España en nombre de Ángel y Agustín Victorero contra los Estados Unidos Mexicanos."

Año: 1932

Estado: Coahuila

AHEEM, CMHMR: MP 142, r. 136, c. 3 .

7. "España en nombre de Ramón García Frade contra los Trstados Unidos Mexicanos."

Año: 1932

Estado: Hidalgo

AHEEM, CMHMR: MP 142, r. 136, c. 3.

8. "España en nombre de Alejo del Cueto contra los Estados Unidos Mexicanos."

Año: 1932

Estado: Coahuila

AHEEM, CMHMR: MP 142, r. 136, c. 3.

9. "España en nombre de Daniel Terán Șáinz contra los Estados Unidos Mexicanos."

Año: 1932

Estado: Durango

AHEEM, CMHMR: MP 142, r. 137, c. 4.

10. "España en nombre de Nicanor Amieva contra los Estados Unidos Mexicanos."

Año: 1932

Estado: Puebla

AHEEM, CMHMR: MP 142, r. 137 , c. 4.

11. "España en nombre de Fernando Monge contra los Estados Unidos Mexicanos."

Año: 1932

Estado: Puebla

AHEEM, CMHMR: MP 142, r. 137, c. 4. 
12. "España en nombre de Antonio y Rafael de la Borbolla contra los Estados Unidos Mexicanos."

Año: 1932

Estado: Morelos

AHEEM, CMHMR: MP 142, r. 137, c. 4.

13. "España en nombre de Evaristo Barbosa contra los Estados Unidos Mexicanos."

Año: 1929

Estado: Tlaxcala

AHEEM, CMHMR: MP 142, r. 137, c. 5.

14. "España en nombre de Miguel Lasso Penagus contra los Estados Unidos Mexicanos."

Año: 1932

Estado: Veracruz

AHEEM, CMHMR: MP 142, r. 137, c. 5.

15. "España en nombre de Julia Pagaza viuda de Alonso contra los Estados Uniclos Mexicanos."

Año: 1932

Estado: Morelos

AHEEM, CMHMR: MP 142, r. 138, c. 6.

16. "España en nombre de Gumersindo Tames Molleda contra los Estados Unidos Mexicanos."

Año: 1932

Estado: Hidalgo

AHEEM, CMHMR: MP 142 , r. 138, c. 6.

17. "España en nombre de José y Agustín González, hoy sus herederos, contra los Estados Unidos Mexicanos." Año: 1932

Estado: Chihuahua

AHEEM, CMHMR: MP 142, r. 138, c. 7.

18. "España en nombre de Inocencio
Narezo, sucesión, contra los Estados Unidos Mexicanos."

Año: 1931

Estado: San Luis Potosí

AHEEM, CMHMR: MP 142, r. 138, c. 7.

19. "España en nombre de Diego Barrena contra los Estados Unidos Mexicanos."

Año: 1930

Estado: Nayarit

AHEEM, CMHMR: MP 142, r. 138, c. 7.

20. "España en nombre de Nazario Sáiz Gaite contra los Estados Unidos Mexicanos."

Año: 1932

Estado: Distrito Federal

AHEEM, CMHMR: MP 142, r. 138, c. 8.

21. "España en nombre de Ramón Fernández contra los Estados Unidos Mexicanos."

Año: 1932

Estado: Puebla

AHEEM, CMHMR: MP 142, r. 138, c. 8.

22. "España en nombre de Manuel Sáinz Pardo contra los Estados Unidos Mexicanos."

Año: 1930

Estado: Veracruz

AHEEM, CMHMR: MP 142, r. 139, c. 8.

23. "España en nombre de Juan Ibarrola contra los Estados Unidos Mexicanos."

Año: s.f.

Estado: Morelos

AHEEM, CMHMR: MP 142, r. 139, c. 8.

24. "España en nombre de Policarpo Suso contra los Estados Unidos Mexicanos." 
Año: 1932

Estado: Durango

AHEEM, CMHMR: MP 142 , r. 139 , c. 8

25. "España en nombre de Andrés Tarrech contra los Estados Unidos Mexicanos."

Año: 1931

Estado: Distrito Federal

AHEEM, CMHMR: MP 142, r. 139, c. 9.

26. "España en nombre de Alfredo Vega contra los Estados Unidos Mexicanos."

Año: 1932

Estado: Tabasco

AHEEM, CMHMR: MP 142, r. 139, c. 9.

27. "España en nombre de J. Suárez y hermano contra los Estados Unidos Mexicanos."

Año: 1932

Estado: Veracruz

AHEEM, CMHMR: MP 142, r. 139, c. 9.

28. "España en nombre de Apolinar Zarain Pereda contra los Estados Unidos Mexicanos."

Año: 1932

Estado: Puebla

AHEEM, CMHMR: MP 142, r. 139, c. 9.

29. "España en nombre de Pablo de la Arena contra los Estados Unidos Mexicanos."

Año: s.f.

Estado:

AHEEM, CMHMR: MP 142, r. 139, c. 9.

30. "España en nombre de Marcial Rodríguez contra los Estados Unidos Mexicanos."

Año: 1930
Estado: Puebla

AHEEM, CMHMR: MP 142, r. 139, c. 10.

31. "España en nombre de Gabino Canales contra los Estados Unidos Mexicanos."

Año: 1932

Estado: Chihuahua

AHEEM, CMHMR: MP 142, r. 140, c. 10.

32. "España en nombre de Lorenzo Baygorri, hoy sus herederos, contra los Estados Unidos Mexicanos."

Año: 1932

Estado: Morelos

AHEEM, CMHMR: MP 142, r. 140, c. 10.

33. "España en nombre de la testamentaría de Tomás Mendirichaga, Mariano Hernández, Ricardo Hernández y Julio Hernández contra los Estados Unidos Mexicanos."

Año: 1932

Estado: Nuevo León

AHEEM, CMHMR: MP 142, r. 140, c. 11.

34. "España en nombre de Antonio y Blas Reguero Pérez contra los Estados Unidos Mexicanos."

Año: 1932

Estado: Puebla

AHEEM, CMHMR: MP 142, r. 140, c. 12.

35. "España en nombre de Ernesto Sánchez del Arenal contra los Estados Unidos Mexicanos."

Año: 1931

Estado: Chiapas

AHEEM, CMHMR: MP 142, r. 141, c. 12.

36. "España en nombre de Pedro del Cueto contra los Estados Unidos Mexicanos." 
Año: 1931

Estado: Chiapas

AHEEM, CMHMR: MP 142, r. 141, c. 12.

37. "España en nombre de José Viñal contra los Estados Unidos Mexicanos." Año: 1932

Estado: Coahuila

AHEEM, CMHMR: MP 142, r. 141, c. 12.

38. "España en nombre de José Santa Coloma contra los Estados Unidos Mexicanos."

Año: 1932

Estado: Guanajuato

AHEEM, CMHMR: MP 142, r. 141, c. 12.

39. "España en nombre de Ramón

Cost Prat contra los Estados Unidos Mexicanos."

Año: 1932

Estado: Distrito Federal

AHEEM, CMHMR: MP 142, r. 141, c. 12.

40. "España en nombre de Antonio

H. Portilla contra los Estados Unidos Mexicanos."

Año: 1930

Estado: Distrito Federal

AHEEM, CMHMR: MP 142, r. 141, c. 13.

41. "España en nombre de Cueto y Cía contra los Estados Unidos Mexicanos." Año: 1932

Estado: Chiapas

AHEEM, CMHMR: MP 142, r. 141, c. 13.

42. "España en nombre de Manuel Reta contra los Estados Unidos Mexicanos."

Año: 1931

Estado: Veracruz

AHEEM, CMHMR: MP 142, r. 141, c. 13.
43. "España en nombre de Serafín . González Antuñano contra los Estados Unidos Mexicanos."

Año: 1932

Estado: Veracruz

AHEEM, CMHMR: MP 142, r. 141, c. 13.

44. "España en nombre de Calixto Márquez contra los Estados Unidos Mexicanos."

Año: 1932

Estado: Veracruz

AHEEM, CMHMR: MP 142, r. 141, c. 13.

45. "España en nombre de Pablo Gutiérrez contra los Estados Unidos Mexicanos.".

Año: 1932

Estado: Durango

AHEEM, CMHMR: MP 142, r. 141, c. 13.

46. "España en nombre de Daniel Revuelta contra los Estados Unidos Mexicanos."

Año: 1932

Estado: Coahuila

AHEEM, CMHMR: MP 142, r. 142, c. 13.

47. "España en nombre de Alejo Arechederra, hoy sus herederos, contra los Estados Unidos Mexicanos."

Año: 1930

Estado: Puebla

AHEEM, CMHMR: MP 142, r. 142, c. 13.

48. "España en nombre de José Ramón Suárez contra los Estados Unidos Mexicanos."

Año: 1932

Estado: Chihuahua

AHEEM, CMHMR: MP 142, r. 142, c. 14.

49. "España en nombre de Constan- 
tino Sobrado Salgueiro contra los Estados Unidos Mexicanos."

Año: s.f.

Estado: Veracruz.

AHEEM, CMHMR: MP 142, r. 142, c. 14.

50. "España en nombre de Maura Robles de San Sebastián contra los Estados Unidos Mexicanos."

Año: 1932

Estado: Chiapas

AHEEM, CMHMR: MP 142, r. 142, c. 14.

51. "España en nombre de Gil González contra los Estados Unidos Mexicanos."

Año: 1932

Estado: Coahuila

AHEEM, CMHMR: MP 142, r. 142, c. 14.

52. "España en nombre de Pio Ochandarena contra los Estados Unidos Mexicanos."

Año: 1932

Estado: Tamaulipas

AIIEEM, CMHMR: MP 142, r. 142, c. 14.

53. "España en nombre de Manuel Bayón contra los Estados Unidos Mexicanos."

Año: 1930

Estado: Veracruz

AHEEM, CMHMR: MP 142, r. 142, c. 14.

54. "España en nombre de Cecilio González contra los Estados Unidos Mexicanos."

Año: 1930

Estado: Coahuila

AHEEM, CMHMR: MP 142, r. 142, c. 14.

55. "España en nombre de Joaquín de la Torre contra los Estados Unidos
Mexicanos."

Año: 1932

Estado: Distrito Federal

AHEEM, CMHMR: MP 142, r. 142, c. 15.

56. "España en nombre de Manuel Vega Vallina contra los Estados Unidos Mexicanos."

Año: 1932

Estado: Guanajuato

AHEEM, CMHMR: MP 142, r. 142, c. 15.

57. "España en nombre de Concepción Noceda viuda de Quintana contra los Estados Unidos Mexicanos."

Año: 1932

Estado: Tamaulipas

AHEEM, CMHMR: MP 142, r. 142, c. 15.

58. "España en nombre de Lope Nuño Gómez contra los Estados Unidos Mexicanos."

Año: 1932

Estado: Coahuila

AIEEM, CMHMR: MP 142, r. 143, c. 15.

59. "España en nombre de Ramón Otero contra los Estados Línidos Me. xicanos."

Año: 1932

Estado: Tamaulipas

AHEEM, CMHMR: MP 142, r. 143, c. 15.

60. "España en nombre de Manuel Garriclo Barros contra los Estados Unidos Mexicanos."

Año: 1930

Estado: Puebla

AHEEM, CMHMR: MP 142, r. 143, c. 15.

61. "España en nombre de Sotres, Sordo y Cía contra los Estados Unidos Mexicanos." 
Año: 1932

Estado: Distrito Federal

AHEEM, CMHMR: MP 142, r. 143, c. 15.

62. "España en nombre de José Poo Fernández contra los Estados Unidos Mexicanos."

Año: 1932

Estado: Chiapas

AHEEM, CMHMR: MP 142, r. 143, c. 15.

63. "España en nombre de J. Villar y Cía. contra los Estados Únidos Mexicanos."

Año: 1932

Estado: Guerrero

AHEEM, CMHMR: MP 142, r. 143, c. 15.

64. "España en nombre de Rosalino Fernández Paradelo, conocido también por $M$. Rodríguez, hoy sus herederos, contra los Estados Unidos Mexicanos."

Año: 1932

Estado: Chiapas

AHEEM, CMHMR: MP 142, r. 143, c. 15.

65. "España en nombre de Josć Pagaza contra los Estados Unidos Mexicanos." Año: 1932

Estado: Morelos

AHEEM, CMHMR: MP 142, r. 143, c. 132.

66. "España en nombre de Ramón de la Fuente contra los Estados Unidos Mexicanos."

Año: 1930

Estado: Puebla

AHEEM, CMHMR: MP 142, r. 144, c. 16.

67. "España en nombre de Ramón Díez de Sollano y María Portillo de Díez de Sollano contra los Estados Uniclos
Mexicanos."

Año: 1932

Estado: Morelos

AHEEM, CMHMR: MP 142, r. 144, c. 16.

68. "España en nombre de G. Díez Fernández contra los Estados Unidos Mexicanos."

Año: 1932

Estado: Veracruz

AIIEEM, CMHMR: MP 142, r. 144, c. 16.

69. "España en nombre de Valentín Fernández R. contra los Estados Unidos Mexicanos."

Año: 1931

Estado: Nuevo León

AHEEM. CMHMR: MP 142, r. 144, c. 17.

70. "España en nombre de Francisco Gutiérrez Illera contra los Estados Unidos Mexicanos."

Año: 1932

Estado: Guanajuato

AHEEM, CMHMR: MP 142, r. 144, c. 17.

71. "España en nombre de Valeriano Ibáñez contra los Estados Unidos Me. xicanos."

Año: 1932

Estado: Yucatán

AHEEM, CMHMR: MP 142, r. 144, c. 17.

72. "España en nombre de Aniceto R. Neira contra los Estados Unidos Mexicanos."

Año: 1932

Estado: Coahuila

AHEEM, CMHMR: MP 142, r. 144, c. 17.

73. "España en nombre de Modesto Díaz contra los Estados Unidos Mexicanos." 
Año: 1932

Estado: Veracruz

AHEEM, CMHMR: MP 142, r. 144, c. 17.

74. "España en nombre de Bernardo H. Romano contra los Estados Unidos Mexicanos."

Año: 1932

Estado: Puebla

AHEEM, CMHMR: MP 142, r. 145, c. 17.

75. "España en nombre de Joaquín Antonio Egoñe contra los Estados Unidos Mexicanos."

Año: 1932

Estado: San Luis Potosí

AHEEM, CMHMR: MP 142, r. 145, c. 17.

76. "España en nombre de Ramón Mandri contra los Estados Unidos Mexicanos."

Año: 1931

Estado: Estado de México

AHEEM, CMHMR: MP 142, r. 145, c. 18.

77. "España en nombre de Manuel Pereyra Vázquez contra los Estados Unidos Mexicanos."

Año: 1932

Estado: Tabasco

AHEEM, CMHMR: MP 142, r. 145, c. 18.

78. "España en nombre de Luciano Rodríguez contra los Estados Unidos Mexicanos."

Año: 1932

Estado: Puebla

AHEEM, CMHMR: MP 142, r. 145, c. 18.

79. "España en nombre de Miguel Pérez García, hoy su sucesión, contra los Estados Unidos Mexicanos."

Año: 1932
Estado: Veracruz

AHEEM, CMHMR: MP 142, r. 145, c. 19.

80. "España en nombre de José Guzmán contra los Estados Unidos Mexicanos."

Año: 1932

Estado: Coahuila

AHEEM, CMHMR: MP 142, r. 145, c. 18.

81. "España en nombre de Emilio Arroyo contra los Estados Unidos Mexicanos."

Año: 1932

Estado: Chihuahua

AHEEM, CMHMR: MP 142 , r. 145 , c. 18.

82. "España en nombre de Benigno de Hoyos, hoy su sucesión, contra los Estados Unidos Mexicanos."

Año: 1932

Estado: Coahuila

AHEEM, CMHMR: MP 142, r. 145, c. 19.

83. "España en nombre de María Pagaza viuda de Carriles contra los Estados Unidos Mexicanos."

Año: 1932

Estado: Morelos

AHEEM, CMHMR: MP 142, r. 145, c. 19.

84. "España en nombre de Marcos Castrezana, hoy sus herederos, contra los Estados Unidos Mexicanos."

Año: 1931

Estado: Puebla

AHEEM, CMHMR: MP 142, r. 145, c. 19.

85. "España en nombre de Manuel y García Pérez y socios de 'Pérez Hermanos' contra los Estados Unidos Mexicanos."

Año: 1932 
Estado: Tamaulipas

AHEEM, CMHMR: MP 142, r. 145, c. 19.

86. "España en nombre de José Camilo

Cerqueiras contra los Estados Unidos Mexicanos."

Año: 1930

Estado: Morelos

AHEEM, CMHMR: MP 142, r. 146, c. 19.

87. "España en nombre de Clementina Núñez y García Reguera contra los Estados Unidos Mexicanos."

Año: 1932

Estado: Distrito Federal

AHEEM, CMHMR: MP 142, r. 146, c. 19.

88. "España en nombre de Ramón

Canales contra los Estados Unidos Mexicanos."

Año: 1932

Estado: Chihuahua

AHEEM, CMHMR: MP 142, r. 146, c. 19.

89. "España en nombre de Víctor de la "Iorre contra los Estados Unidos Mexicanos."

Año: 1932

Estado: Tamaulipas

AHEEM, CMHMR: MP 142, r. 146, c. 19.

90. "España en nombre de Ricardo

Quintana contra los Estados Unidos Mexicanos."

Año: 1932

Estado: Coahuila

AHEEM, CMHMR: MP 142, r. 146, c. 19.

91. "España en nombre de Cesáreo

J. Martín contra los Estados Unidos Mexicanos."

Año: 1930

Estado: Chihuahua

AHEEM, CMHMR: MP 142, r. 146, c. 20.
92. "España en nombre de Jacinto del Río contra los Estados Unidos Mexicanos."

Año: 1932

Estado: Morelos

AHEEM, CMHMR: MP 142, r. 146, c. 20.

93. "España en nombre de Casimiro Juliá Ferrer contra los Estados Unidos Mexicanos."

Año: 1932

Estado: Chihuahua

AHEEM, CMHMR: MP 142, r. 146, c. 20.

94. "España en nombre de Bernardo Suárez Orejas contra los Estados Unidos Mexicanos."

Año: 1932

Estado: Distrito Federal

AHEEM, CMHMR: MP 142, r. 146, c. 20.

95. "España en nombre de Francisco G. Núñez contra los Estados Unidos Mexicanos."

Año: 1931

Estado: Chihuahua

AHEEM, CMHMR: MP 142, r. 146, c. 20.

96. "España en nombre de Isidoro Gutiérrez contra los Estados Unidos Mexicanos."

Año: 1932

Estado: Chihuahua

AHEEM, CMHMR: MP 142, r. 146, c. 20.

97. "España en nombre de Martín G. Gutiérrez contra los Estados Unidos Mexicanos."

Año: 1932

Estado: Chihuahua

AHEEM, CMHMR: MP 142, r. 147, c. 20.

98. "España en nombre de Ángel Ruiz Ocejo contra los Estados Unidos Mexi- 
canos."

Año: 1931

Estado: Guanajuato

AJIEEM, CMHMR: MP 142, r. 147, c. 20.

99. "España en nombre de Cristóbal Álvarez M. contra los Estados Unidos Mexicanos."

Año: 1932

Estado: Morelos

AHEEM, CMHMR: MP 142, r. 147, c. 21.

100. "España en nombre de José Arruñada contra los Estados Uniclos Mexicanos."

Año: 1932

Estado: Durango

ALEEM, CMHMR: MP 142, r. 147, c. 21.

101. "España en nombre de Dositeo Abella contra los Estados Unidos Mexicanos."

Año: 1930

Estado: Tabasco

AHEEM, CMHMR: MP 142, r. 147, c. 21.

102. "España en nombre de Domingo Moreno y Felipa Busto de Moreno contra los Estados Unidos Mexicanos." Año: 1931

Estado: Tamaulipas

AHEEM, CMHMR: MP 142, r. 147, c. 21.

103. "España en nombre de Julio Gallego, hoy sus herederos, contra los Estados Unidos Mexicanos."

Año: 1932

Estado: Distrito Federal

AHIEEM, CMHMR: MP 142, r. 147, c. 22.

104. "España en nombre de Manuel Rodríguez contra los Estados Unidos Mexicanos."

Año: 1932
Estado: Tabasco

AHEEM, CMHMR: MP 142, r. 148, c. 22.

105. "España en nombre de Pablo Platón contra los Estados Unidos Mexicanos."

Año: s.f.

Estado: Chihuahua

AHEEM, CMHMR: MP 142, r. 148, c. 22.

106. "España en nombre de Marcos Illades contra los Estados Unidos Mexicanos."

Año: 1930

Estado: Guanajuato

AIIEEM, CMHMR: MP 142, r. 148, c. 22.

107. "España en nombre de Miguel Ruiz contra los Estados Unidos Mexicanos."

Año: 1932

Estado: Chihuahua

AHEEM, CMHMR: MP 142, r. 148, c. 22.

108. "España en nombre de Mariano Sagarminaga contra los Estados Unidos Mexicanos."

Año: 1932

Estado: Chihuahua

AHEEM, CMHMR: MP 142, r. 148, c. 22.

109. "España en nombre de Francisco Sáinz contra los Estados Unidos Mexicanos."

Año: 1932

Estado: Chihuahua

AHEEM, CMHMR: MP 142, r. 148, c. 22.

110. "España en nombre de Ángel Mijares contra los Estados Unidos Mexicanos."

Año: 1929

Estado: Distrito Federal

AHEEM, CMHMR: MP 142, r. 148, c. 22. 
111. "España en nombre de Saturnino Llera contra los Estados Unidos Mexicanos."

Año: 1932

Estado: Morelos

AHEEM, CMHMR: MP 142, r. 148, c. 22.

112. "España en nombre de Francisco Trespalacios contra los Estados Unidos Mexicanos."

Año: 1932

Estado: Guerrero

AHEEM, CMHMR: MP 142, r. 148, c. 22.

113. "España en nombre de Manuel Trueba contra los Estados Unidos Mexicanos."

Año: 1930

Estado: Chihuahua

AHEEM, CMHMR: MP 142, r. 148, c. 22.

114. "España en nombre de Francisco Noriega contra los Estados Unidos Mexicanos."

Año: 1932

Estado: Chihuahua

AHEEM, CMHMR: MP 142, r. 148, c. 23.

115. "España en nombre de Francisco y Eugenio Noriega Villar y Matías Celada contra los Estados Unidos Mexicanos."

Año: 1932

Estado: Chihuahua

AHIEM, CMHMR: MP .142, r. 148, c. 23.

116. "España en nombre de José Díaz Casares, hoy su sucesión, contra los Estados Unidos Mexicanos."

Año: 1932

Estado: Nuevo León

AHIEM, CMHMR: MP 142, r. 149, c. 23.

117. "España en nombre de Facundo
Martínez y José Fernández contra los Estados Unidos Mexicanos."

Año: 1932

Estado: Chihuahua

AHEEM, CMHMR: MP 142, r. 149, c. 23.

118. "España en nombre de Juan y Enrique Artasánchez contra los Estados Unidos Mexicanos."

Año: 1932

Estado: Puebla

AHEEM, CMHMR: MP 142 , r. 149 , c. 24.

119. "España en nombre de Antonia Asuria viuda de Romano contra los Estados Unidos Mexicanos."

Año: 1932

Estado: Puebla

AHEEM, CMHMR: MP 142, r. 149, c. 24.

120. "España en nombre de José María Zunzunegui, hoy sus herederos, contra los Estados Unidos Mexicanos."

Año: 1931

Estado: Puebla

AHEEM, CMHMR: MP 142, r. 149, c. 24.

121. "España en nombre de Pedro Tresgallo contra los Estados Unidos Mexicanos."

Año: 1930

Estado: Veracruz

AHEEM, CMHMR: MP 142, r. 149, c. 24.

122. "España en nombre de Manuel Fernández Suárez contra los Estados Unidos Mexicanos."

Año: 1931

Estado: Veracruz

AHEEM, CMHMR: MP 142, r. 150, c. 24.

123. "España en nombre de Marcelino Horgado contra los Estados Unidos Mexicanos." 
Año: 1932

Estado: Coahuila

AHEEM, CMHMR: MP 142, r. 150, c. 25.

124. "España en nombre de Gregorio Iparraguirre contra los Estados Unidos Mexicanos."

Año: 1930

Estado: Guanajuato

AHEEM, CMHMR: MP 142, r. 150, c. 25.

125. "España en nombre de Rosendo Sordo Noriega contra los Estados Unidos Mexicanos."

Año: 1932

Estado: Puebla

AHEEM, CMHMR: MP 142, r. 150, c. 25.

126. "España en nombre de Julián Iturralde y García contra los Estados Unidos Mexicanos."

Año: 1932

Estado: Durango

AHEEM, CMHMR: MP 142, r. 150, c. 25.

127. "España en nombre de Gaspar Díaz contra los Estados Unidos Mexicanos."

Año: 1932

Estado: Guanajuato

AHEEM, CMHMR: MP 142, r. 150, c. 25.

128. "España en nombre de Vicente Vidal, hoy sus herederos, contra los Estados Unidos Mexicanos."

Año: 1930

Estado: Tamaulipas

AHEEM, CMHMR: MP 142, r. 150, c. 25.

129. "España en nombre de Félix Solana contra los Estados Unidos Mexicanos."

Año: s.f.

Estado: Oaxaca

AHEEM, CMHMR: MP 142, r. 150, c. 25.
130. "España en nombre de Gonzalo Piñón y herederos de Pedro Piñón contra los Estados Unidos Mexicanos." Año: 1932

Estado: Coahuila

AHEEM, CMHMR: MP 142, r. 150, c. 25.

131. "España en nombre de Juan Bilbao contra los Estados Unidos Mexicanos."

Año: 1932

Estado: Chihuahua

AHEEM, CMHMR: MP 142, r. 150, c. 26.

132. "España en nombre de Enrique Garabana contra los Estados Unidos Mexicanos."

Año: 1932

Estado: Quintana Roo

AHEEM, CMHMR: MP 142, r. 150, c. 26.

133. "España en nombre de Anastasio Gutiérrez Torre contra los Estados Unidos Mexicanos."

Año: 1932

Estado: Veracruz

AHEEM, CMHMR: MP 142, r. 150, c. 26.

134. "España en nombre de Manuel Lapuente, hoy sus herederos, contra los Estados Unidos Mexicanos."

Año: 1932

Estado: Puebla

AHEEM, CMHMR: MP 142, r. 151, c. 26.

135. "España en nombre de Tomás Sierra, sucesión, contra los Estados Unidos Mexicanos."

Año: 1932

Estado: Guerrero

AHEEM, CMHMR: MP 142, r. 151, c. 26.

136. "España en nombre de Genaro Mere y Pando, hoy sus herederos, 
contra los Estados Unidos Mexicanos." Año: 1932

Estado: Chiapas

AHEEM, CMHMR: MP 142, r. 151, c. 26.

137. "España en nombre de Ramón

Sierra Pando contra los Estados Unidos Mexicanos."

Año: 1932

Estado: Guerrero

AHEEM, CMHMR: MP 142, r. 151, c. 26.

138. "España en nombre de Eusebio Gómez Lavín contra los Estados Unidos Mexicanos."

Año: 1931

Estado: Yucatán

AHEEM, CMHMR: MP 142, r. 151, c. 26.

139. "España en nombre de Pedro Cobo, hoy sus herederos, contra los Estados Unidos Mexicanos."

Año: 1932

Estado: San Luis Potosí

AHEEM, CMHMR: MP 142, r. 151, c. 27.

140. "España en nombre de Ramón Urdapilleta, hoy sus herederos, contra los Estados Unidos Mexicanos."

Año: 1932

Estado: Estado de México

AHEEM, CMHMR: MP 142, r. 151, c. 27.

141. "España en nombre de Rogelio Gómez y hermano contra los Estados Unidos Mexicanos."

Año: 1932

Estado: Oaxaca

AHEEM, CMHMR: MP 142, r. 151, c. 27.

142. "España en nombre de José Miguel Hurtado contra los Estados Unidos Mexicanos."

Año: 1931
Estado: Coahuila

AHEEM, CMHMR: MP 142, r. 151, c. 27.

143. "España en nombre de Valentín Gutiérrez García contra los Estados Unidos Mexicanos."

Año: 1932

Estado: Distrito Federal

AHEEM, CMHMR: MP 142, r. 151, c. 27.

144. "España en nombre de Dolores Arce viuda de Aguirre, hoy sus herederos, contra los Estados Unidos Mexicanos."

Año: s.f.

Estado: Zacatecas

AHEEM, CMHMR: MP 142, r. 151, c. 27.

145. "España en nombre de Ricardo Villa contra los Estados Unidos Mexicanos."

Año: 1931

Estado: Veracruz

AHEEM, CMHMR: MP 142, r. 151, c. 27.

146. "España en nombre de José Verdaguer contra los Estados Unidos Mexicanos."

Año: Distrito Federal

Estado: 1932

AHEEM, CMHMR: MP 142, r. 152, c. 27.

147. "España en nombre de la sucesión de Laureano Negrete contra los Estados Unidos Mexicanos."

Año: s.f.

Estado: Estado de México

AHEEM, CMHMR: MP 142, r. 152, c. 27.

148. "España en nombre de Francisco Orquín contra los Estados Unidos Mexicanos."

Año: 1932

Estado: Durango

AHEEM, CMHMR: MP 142, r. 152, c. 28. 
149. "España en nombre de Pedro Vallado contra los Estados Unidos Mexicanos."

Año: 1932

Estado: Distrito Federal

AHEEM, CMHMR: MP 142, r. 152, c. 28.

150. "España en nombre de Carmen Vives contra los Estados Unidos Mexicanos."

Año: 1930

Estado: Oaxaca

AHEEM, CMHMR: MP 142, r. 152, c. 28.

151. "España en nombre de José Gorbeña contra los Estados Unidos Mexicanos."

Año: 1932

Estado: Guanajuato

AHEEM, CMHMR: MP 142, r. 152, c. 28.

152. "España en nombre de Pablo Díaz O. contra los Estados Unidos Mexicanos."

Año: 1932

Estado: Tabasco

AHEEM, CMHMR: MP 142, r. 152, c. 28.

153. "España en nombre de Eulogio Fernánclez contra los Estados Unidos Mexicanos."

Año: 1932

Estado: Coahuila

AHEEM, CMHMR: MP 142, r. 152, c. 28.

154. "España en nombre de Manuel Gómez y hermano contra los Estados Unidos Mexicanos."

Año: 1932

Estado: Guanajuato

AIIEEM, CMHMR: MP 142, r. 152, c. 28.

155. "España en nombre de Valentín Gómez, cesionario de Gómez y Ríos, contra los Estados Unidos Mexicanos."

Año: 1932

Estado: Tlaxcala

AHEEM, CMHMR: MP 142, r. 152, c. 28.

156. "España en nombre de Leopoldo Vial y Henly contra los Estados Unidos Mexicanos."

Año: 1932

Estado: Veracruz

AHEEM. CMHMR: MP 142, r. 152, c. 29.

157. "España en nombre de Catalina Vázquez Parada viuda de Zarandona contra los Estados Unjdos Mexicanos." Año: 1932

Estado: Guanajuato

AHEEM, CMHMR: MP 142, r. 153, c. 29.

158. "España en nombre de Tomás Sordo contra los Estados Unidos Mexicanos."

Año: 1932

Estado: Morelos

AHFEM, CMHMR: MP 142, r. 153, c. 29.

159. "España en nombre de Ramón Vázquez contra los Estados Unidos Mexicanos."

Año: 1932

Estado: Guanajuato

AHEEM, CMHMR: MP 142, r. 153, c. 29.

160. "España en nombre de Julián Romano Villar, hoy sus herederos, contra los Estados Unidos Mexicanos." Año: 1932

Estado: Guerrero

AHEEM, CMHMR: MP 142, r. 153, c. 29.

161. "España en nombre de Federico Rodríguez contra los Estados Unidos Mexicanos."

Año: 1932 
Estado: Chiapas

AHEEM, CMHMR: MP 142, r. 153, c. 29.

162. "España en nombre de Martín Sañudo, hoy su sucesión, contra los Estados Unídos Mexicanos."

Año: 1932

Estado: Guanajuato

AHEEM, CMHMR: MP 142, r. 153, c. 29.

163. "España en nombre de José Gutiérrez, hoy sus herederos, contra los Estados Unidos Mexicanos."

Año: 1932

Estado: Durango

AHEEM, CMHMR: MP 142, r. 153, c. 29.

164. "España en nombre de Julián Romano y Cía. contra los Estados Unidos Mexicanos."

Año: 1932

Estado: Guerrero

AHEEM, CMHMR: MP 142, r. 153, c. 29.

165. "España en nombre de Testamentaría de Enrique Soto Cortina contra los Estados Unidos Mexicanos."

Año: 1932

Estado: Morelos

AHEEM, CMHMR: MP 142, r. 153, c. 29.

166. "España en nombre de Ramón Ibargüengoitia, hoy sus herederos, contra los Estados Unidos Mexicanos." Año: 1932

Estado: San Luis Potosí

AHEEM, CMHMR: MP 142, r. 153, c. 29.

167. "España en nombre de Ignacio Fernández contra los Estados Unidos Mexicanos."

Año: 1932

Estado: San Luis Potosí

AHEEM, CMHMR: MP 142, r. 153, c. 29.
168. "España en nombre de Francisco V. Ibargüengoitia contra los Estados Unidos Mexicanos."

Año: 1932

Estado: Tamaulipas

AHEEM, CMHMR: MP 142, r. 153, c. 30.

169. "España en nombre de Óscar Ocharán contra los Estados Unidos Mexicanos."

Año: s.f.

Estado: Sonora

AHEEM, CMHMR: MP 142, r. 154, c. 30.

170. "España en nombre de Álvaro González contra los Estados Unidos Mexicanos."

Año: 1932

Estado: Coahuila

AHEEM, CMHMR: MP 142, r. 154, c. 30.

171. "España en nombre de Ambrosio Sampedro contra los Estados Unidos Mexicanos."

Año: 1932

Estado: Durango

AHEEM, CMHMR: MP 142, r. 154, c. 31.

172. "España en nombre de Miguel Echeverría contra los Estados Unidos Mexicanos."

Año: 1932

Estado: Coahuila

AHEEM, CMHMR: MP 142, r. 154, c. 31.

173. "España en nombre de Enrique Zavala y Manuela Muriedas de Zavala contra los Estados Unidos Mexicanos." Año: 1931

Estado: San Luis Potosí

AHEEM, CMHMR: MP 142, r. 154, c. 32.

174. "España en nombre de Nicolás Alonso contra los Estados Unidos Me- 
xicanos."

Año: 1931

Estado: Distrito Federal

AHEEM, CMHMR: MP 142, r. 154, c. 31.

175. "España en nombre de Benito Arias Gómez contra los Estados Unidos Mexicanos."

Año: 1932

Estado: Puebla

AHEEM, CMHMR: MP 142, r. 154, c. 32.

176. "España en nombre de Venancio Artola y Usaola contra los Estados Unidos Mexicanos."

Año: 1931

Estado: Distrito Federal

AHEEM, CMHMR: MP 142, r. 155, c. 32.

177. "España en nombre de Braulio Agudo contra los Estados Unidos Mexicanos."

Año: 1932

Estado: Distrito Federal

AHEEM, CMHMR: MP 142, r. 155, c. 32.

178. "España en nombre de Domingo Abuin contra los Estados Unidos Mexicanos."

Año: s.f.

Estado: Distrito Federal

AHEEM, CMHMR: MP 142, r. 155, c. 32.

179. "España en nombre de Rafael Alonso contra los Estados Unidos Mexicanos."

Año: 1932

Estado: Morelos

AHEEM, CMHMR: MP 142, r. 155, c. 32.

180. "España en nombre de Francisco Areces, sucèsión, contra los Estados Unidos Mexicanos."

Año: 1932
Estado: Veracruz

AHEEM, CMHMR: MP 142, r. 155, c. 32.

181. "España en nombre de Francisco Alonso contra los Estados Unidos Mexicanos."

Año: 1932

Estado: Puebla

AHEEM, CMHMR: MP 142, r. 155, c. 32.

182. "España en nombre de Juan Amieva contra los Estados Unidos Mexicanos."

Año: s.f.

Estado: Oaxaca

AHEEM, CMHMR: MP 142, r. 155, c. 32.

183. "España en nombre de César Alebo contra los Estados Unidos Mexicanos."

Año: 1930

Estado: San Luis Potosí

AHEEM, CMHMR: MP 142, r. 155, c. 33.

184."España en nombre de Ildefonsa Arnesto viuda de Aranda contra los Estados Unidos Mexicanos."

Año: 1932

Estado: Oaxaca

AHEEM, CMHMR: MP 142, r. 155, c. 33.

185. "España en nombre de Eladio Amor contra los Estados Unidos Mexicanos."

Año: 1931

Estado: Tamaulipas

AHEEM, CMHMR: MP 142, r. 155, c. 33.

186. "España en nombre de Manuel Arechavala contra los Estados Unidos Mexicanos."

Año: 1932

Estado: Estado de México

AHEEM, CMHMR: MP 142, r. 155, c. 33. 
187. "España en nombre de Isidro Allende Piñuela contra los Estados Unidos Mexicanos."

Año: 1932

Estado: Guanajuato

AHEEM, CMHMR: MP 142, r. 155, c. 33.

188. "España en nombre de Andrés Ahedo contra los Estados Unidos Mexicanos."

Año: 1930

Estadio: Estado de México

AHEEM, CMHMR: MP 142, r. 156, c. 34.

189. "España en nombre de Benigno Álvarez Rodríguez contra los Estados Unidos Mexicanos."

Año: 1932

Estado: Chiapas

AHEEM, CMHMR: MP 142, r. 156, c. 34.

190. "España en nombre de Ramón y Martín Arias contra los Estados Unidos Mexicanos."

Año: 1932

Estado: Estado de México

AHEEM, CMHMR: MP 142, r. 156, c. 34.

191. "España en nombre de José Arboleya contra los Estados Unidos Mexicanos."

Año: 1931

Estado: Estado de México

AHEEM, CMHMR: MP 142, r. 156, c. 34.

192. "España en nombre de José Pría Álvarez contra los Estados Unidos Mexicanos."

Año: 1932

Estado: Distrito Federal

AHEEM, CMHMR: MP 142, r. 156, c. 34.

193. "España en nombre de Cruz Bustos contra los Estados Unidos Mexicanos."
Año: 1932

Estado: Tabasco

AHEEM, CMHMR: MP 142, r. 156, c. 34.

194. "España en nombre de Pedro Bárcena Trueba contra los Estados Unidos Mexicanos."

Año: 1932

Estado: Nuevo León

AHEEM, CMHMR: MP 142, r. 156, c. 34 .

195. "España en nombre de Porfirio Bárcena y herederos de Santos Bárcena contra los Estados Unidos Mexicanos."

Año: 1932

Estado: Distrito Federal

AHEEM, CMHMR: MP 142, r. 156, c. 34.

196. "España en nombre de Manuel Berriozábal contra los Estados Unidos Mexicanos."

Año: 1932

Estado: Distrito Federal

AHEEM, CMHMR: MP 142, r. 156, c. 35.

197. "España en nombre de Luis Bermejillo, hoy su sucesión, contra los Estados Unidos Mexicanos."

Año: 1932

Estado: Guanajuato

AHEEM, CMHMR: MP 142, r. 156, c. 35.

198. "España en nombre de Francisco Bellas contra los Estados Unidos Mexicanos."

Año: 1930

Estado: Distrito Federal

AHEEM, CMHMR: MP 142, r. 157, c. 35.

199. "España en nombre de Juan y Francisco Bueno contra los Estados Unidos Mexicanos."

Año: 1932 
Estado: Oaxaca

AHEEM, CMHMR: MP 142, r. 157, c. 36.

200. "España en nombre de Antonio Galdoros contra los Estados Unidos Mexicanos."

Año: s.f.

Estado: Distrito Federal

AHEEM, CMHMR: MP 142, r. 157, c. 36.

201. "España en nombre de Antonio Morell, Ángel Pérez Olivares y herederos de Gregorio Benito, todos como socios de la 'Sociedad G. Benito y Cía. Sucursales' contra los Estados Unidos Mexicanos."

Año: 1932

Estado: Tabasco

AHEEM, CMHMR: MP 142, r. 157, c. 36.

202. "España en nombre de Felia Barandiarán contra los Estados Unidos Mexicanos."

Año: 1932

Estado: Puebla

AHEEM, CMHMR: MP 142, r. 157, c. 36.

203. "España en nombre de Casimiro Balanzategui contra los Estados Unidos Mexicanos."

Año: 1932

Estado: Estado de México

AHEEM, CMHMR: MP 142, r. 157, c. 36.

204. "España en nombre de Francisco Bardán, sucesión, contra los Estados Unidos Mexicanos."

Año: 1932

Estado: Coahuila

AHEEM, CMHMR: MP 142, r. 158, c. 36.

205. "España en nombre de Antonio y Manuel Bustillo del Canto contra los
Estados Unidos Mexicanos."

Año: 1932

Estado: Morelos

AHEEM, CMHMR: MP 142, r. 158, c. 36.

206. “España en nombre de Francisco Beridi, hoy sus herederos, contra los Estados Unidos Mexicanos."

Año: 1932

Estado: Morelos

AHEEM, CMHMR: MP 142, r. 158, c. 37.

207. "España en nombre de Antonio Brunet Gelabert contra los Estados Unidos Mexicanos."

Año: 1932

Estado: Veracruz

AHEEM, CMHMR: MP 142, r. 158, c. 37.

208. "España en nombre de Antonio Bonet Ferrer contra los Estados Unjdos Mexicanos."

Año: 1932

Estado: Querétaro

AHEEM, CMHMR: MP 142, r. 158, c. 37.

209. "España en nombre de Prudencio Colsa contra los Estados Unidos Mexicanos."

Año: 1932

Estado: Distrito Federal

AHEEM, CMHMR: MP 142, r. 158, c. 37.

210. "España en nombre de Alfredo Cano contra los Estados Unidos Mexicanos."

Año: 1932

Estado: Veracruz

AHEEM, CMHMR: MP 142, r. 158, c. 37.

211. "España en nombre de Gumersindo Capilla, como único representante de la 'Sociedad Capilla Herma- 
nos', contra los Estados Unidos Mexicanos."

Año: 1932

Estado: Distrito Federal

AHEEM, CMHMR: MP 142, r. 158, c. 37.

212. "España en nombre de Romualdo del Campo contra los Estados Unidos Mexicanos."

Año: 1932

Estado: San Luis Potosí

AHEEM, CMHMR: MP 142, r. 158, c. 37.

213. "España en nombre de Eustaquio de Cos contra los Estados Unidos Mexicanos."

Año: 1930

Estado: San Luis Potosí

AHEEM, CMHMR: MP 142, r. 158, c. 37.

214. "España en nombre de Juan Castillo López contra los Estados Unidos Mexicanos."

Año: 1931

Estado: Durango

AHEEM, CMHMR: MP 142, r. 158, c. 38.

215. "España en nombre de Antonio de la Concha y Francisco de P. Diaque contra los Estados Unidos Mexicanos." Año: 1932

Estado: San Luis Potosí

AHEEM, CMHMR: MP 142, r. 158, c. 38.

216. "España en nombre de Alberto Castaño Marqués, hoy sus herederos, contra los Estados Unidos Mexicanos." Año: 1932

Estado: Durango

AHEEM, CMHMR: MP 142, r. 159, c. 38.

217. "España en nombre de Pablo Carranza Angulo, hoy sus herederos, contra los Estados Unidos Mexicanos." Año: 1932

Estado: Durango/Coahuila

AHEEM, CMHMR: MP 142, r. 159, c. 38.

218. "España en nombre de Matilde Cervantes viuda de De la Horga contra los Estados Unidos Mexicanos."

Año: 1932

Estado: Hidalgo

AHEEM, CMHMR: MP 142, r. 159, c. 38.

219. "España en nombre de Alfonso

C. Canalda contra los Estados Unidos Mexicanos."

Año: 1932

Estado: Chihuahua

AHEEM, CMHMR: MP 142, r. 159, c. 38.

220. "España en nombre de Antonio Conde contra los Estados Unidos Mexicanos."

Año: 1932

Estado: Tamaulipas

AHEEM, CMHMR: MP 142, r. 159, c. 38.

221. "España en nombre de Gerónimo Campos Cabezas contra los Estados Unidos Mexicanos."

Año: 1930

Estado: Estado de México

AHEEM, CMHMR: MP 142, r. 159, c. 39.

222. "España en nombre de Antonio Concepción contra los Estados Unidos Mexicanos."

Año: 1932

Estado: Puebla

AHEEM, CMHMR: MP 142, r. 159, c. 39.

223. "España en nombre de Juan Bautista Ciganda y herederos de Carlos Ciganda contra los Estados Unidos Mexicanos." 
Año: 1932

Estado: Guerrero

AHEEM, CMHMR: MP 142, r. 159, c. 39.

224. "España en nombre de Indalecio Díaz Mendoza contra los Estados Unidos Mexicanos."

Año: 1932

Estado: Guanajuato

AHEEM, CMHMR: MP 142, r. 159, c. 39.

225. "España en nombre de Felipe Cuevas contra los Estados Unidos Mexicanos."

Año: 1930

Estado: Estado de México

AHEEM, CMHMR: MP 142, r. 159, c. 39.

226. "España en nombre de Visitación

Celada de Berenguer contra los Estados Unidos Mexicanos."

Año: 1932

Estado: Coahuila

AHEEM, CMHMR: MP 142, r. 160, c. 39.

227. "España en nombre de José Díaz contra los Estados Unidos Mexicanos." Año: 1931

Estado: Estado de México

AHEEM, CMHMR: MP 142, r. 160, c. 40.

228. "España en nombre de Aurelio Devesa, hoy sus sucesores, contra los Estados Unidos Mexicanos."

Año: 1932

Estado: Distrito Federal

AHEEM, CMHMR: MP 142, r. 160, c. 40.

229. "España en nombre de Francisco Díez González y Guadalupe de Díez González contra los Estados Unidos Mexicanos."

Año: 1931
Estado: Durango

AHEEM, CMHMR: MP 142, r. 160, c. 40.

230. "España en nombre de Jesús Díez Fernández contra los Estados Unidos Mexicanos."

Año: 1931

Estado: Veracruz

AHEEM, CMHMR: MP 142, r. 160, c. 40.

231. "España en nombre de Vicente Deya Arbona contra los Estados Unidos Mexicanos."

Año: 1931

Estado: Chiapas

AHEEM, CMHMR: MP 142, r. 160 , c. 40.

232. "España en nombre de Juan C. Echandi contra los Estados Unidos Mexicanos."

Año: 1932

Estado: Sonora

AHEEM, CMHMR: MP 142, r. 160, C. 40.

233. "España en nombre de Andrés Fernández Alonso, hoy sus herederos, contra los Estados Unidos Mexicanos." Año: 1932

Estado: Puebla

AHEEM, CMHMR: MP 142, r. 160, c. 40.

234. "España en nombre de Ramón Fernández contra los Estados Unidos Mexicanos."

Año: 1931

Estado: Distrito Federal

AHEEM, CMHMR: MP 142, r. 160, c. 40.

235. "España en nombre de Sergio Fernández, hoy sus herederos, contra los Estados Unidos Mexicanos."

Año: 1931

Estado: Coahuila

AHEEM, CMHMR: MP 142, r. 160, c. 41. 
236. "España en nombre de Rafael Castiello, albacea de la testamentaría de Josefa Martínez Negrete viuda de Fernández del Valle, contra los Estados Unidos Mexicanos."

Año: 1932

Estado: Michoacán/Jalisco

AHEEM, CMHMR: MP 142, r. 160, c. 41.

237. "España en nombre de Juan José Rosillo contra los Estados Unidos Mexicanos."

Año: 1932

Estado: Coahuila

AHEEM, CMHMR: MP 142, r. 160, C. 41.

238. "España en nombre de Martín Fernández Tascón contra los Estados Unidos Mexicanos."

Año: 1932

Estado: Durango

AHEEM, CMHMR: MP 142, r. 160, c. 41.

239. "España en nombre de Dámaso Lombrera Ruiz contra los Estados Unidos Mexicanos."

Año: 1932

Estado: Chihuahua

AHEEM, CMHMR: MP 142, r. 161, c. 41.

240. "España en nombre de Alfredo Fernández Flores contra los Estados Unidos Mexicanos."

Año: 1932

Estado: Estado de México

AHEEM, CMHMR: MP 142, r. 161, c. 41.

241. "España en nombre de Casto de la Fuente Parrés contra los Estados Unidos Mexicanos."

Año: 1932

Estado: Estado de México

AHEEM, CMHMR: MP 142, r. 161, c. 41.
242. "España en nombre de Baldomero Freixanet contra los Estados Unidos Mexicanos."

Año: 1932

Estado: Tlaxcala

AHEEM, CMHMR: MP 142, r. 161, c. 41.

243. "España en nombre de Segundino F. Hevia contra los Estados Unidos Mexicanos."

Año: 1930

Estado: Chihuahua

AHEEM, CMHMR: MP 142, r. 161, c. 42.

244. "España en nombre de Demetrio Fagoaga contra los Estados Unidos Mexicanos."

Año: 1931

Estado: Distrito Federal

AHEEM, CMHMR: MP 142, r. 161, c. 43.

245. "España en nombre de Francisco Codinach, sucesión, contra los Estados Unidos Mexicanos."

Año: 1932

Estado: Oaxaca

AHEEM, CMHMR: MP 142, r. 161, c. 43.

246. "España en nombre de Pedro Fernández contra los Estados Unidos Mexicanos."

Año: 1932

Estado: Distrito Federal

AHEEM, CMHMR: MP 142, r. 161, c. 43.

247. "España en nombre de Rafaela Almada viuda de De la Vega, hoy su heredera, contra los Estados Unidos Mexicanos."

Año: 1932

Estado: Sinaloa

AHEEM, CMHMR: MP 142, r. 161, c. 43. 
248. "España en nombre de Manuel Gutiérrez contra los Estados Unidos Mexicanos."

Año: 1932

Estado: Durango

AHEEM, CMHMR: MP 142, r. 162, c. 43.

249. "España en nombre de José González Alonso contra los Estados Unidos Mexicanos."

Año: 1932

Estado: Distrito Federal

AHEEM, CMHMR: MP 142, r. 162, c. 44.

250. "España en nombre de Ezequiel González contra los Estados Unidos Mexicanos."

Año: 1932

Estado: Distrito Federal

AHEEM, CMHMR: MP 142, r. 162, c. 44.

251. "España en nombre de Félix González, hoy su sucesión, contra los Estados Unidos Mexicanos."

Año: 1932

Estado: Distrito Federal

AHEEM, CMHMR: MP 142, r. 162, c. 44.

252. "España en nombre de Pedro González contra los Estados Unidos Mexicanos."

Año: s.f.

Estado: Estado de México

AHEEM, CMHMR: MP 142, r. 162, c. 44.

253. "España en nombre de Joaquín Gutiérrez Bordoy contra los Estados Unidos Mexicanos."

Año: 1932

Estado: Puebla

AHEEM, CMHMR: MP 142, r. 162, c. 44.

254. "España en nombre de Filemón
González contra los Estados Unidos Mexicanos."

Año: 1931

Estado: Distrito Federal

AHEEM, CMHMR: MP 142, r. 162, c. 44.

255. "España en nombre de Alfonso Garrido contra los Estados Unidos Mexicanos."

Año: 1931

Estado: Nuevo León

AHEEM, CMHMR: MP 142, r. 162, c. 45.

256. "España en nombre de Celestino González, sucesión, contra los Estados Unidos Mexicanos."

Año: 1932

Estado: Veracruz

AHEEM, CMHMR: MP 142, r. 162, C. 45.

257. "España en nombre de Eduardo Torre Tolnado, sucesión, contra los Estados Unidos Mexicanos."

Año: 1932

Estado: Zacatecas

AHEEM, CMHMR: MP 142, r. 162, C. 45.

258. "España en nombre de Bernardino Torre Tolnado contra los Estados Unidos Mexicanos."

Año: 1932

Estado: Coahuila

AHEEM, CMHMR: MP 142, r. 163 , c. 46.

259. "España en nombre de Vicente González contra los Estados Unidos Mexicanos."

Año: 1931

Estado: Puebla

AHEEM, CMHMR: MP 142, r. 163, c. 46.

260. "España en nombre de Isidro Garabana contra los Estados Unidos 
Mexicanos."

Año: 1932

Estado: Quintana Roo

AHEEM, CMHMR: MP 142, r. 163, c. 47.

261. "España en nombre de Félix e Ignacio Monzón contra los Estados Unidos Mexicanos."

Año: 1932

Estado: San Luis Potosí

AHEEM, CMHMR: MP 142, r. 163, c. 47.

262. "España en nombre de Zenón González contra los Estados Unidos Mexicanos."

Año: 1930

Estado: Morelos

AHEEM, CMHMR: MP 142, r. 164, c. 48.

263. "España en nombre de Juan Gómez Merino contra los Estados Unidos Mexicanos."

Año: 1932

Estado: Zacatecas

AHEEM, CMHMR: MP 142, r. 164, c. 48.

264. "España en nombre de Manuel Galvarriato contra los Estados Unidos Mexicanos."

Año: 1932

Estado: Nayarit

AHEEM, CMHMR: MP 142, r. 164, c. 48.

265. "España en nombre de Ramón González Rodríguez, hoy sus herederos, contra los Estados Unidos Mexicanos."

Año: 1932

Estado: Jalisco

AHEEM, CMHMR: MP 142, r. 164, c. 48.

266. "España en nombre de Anacleto García contra los Estados Unidos Mexicanos."
Año: s.f.

Estado: Durango

AHEEM, CMHMR: MP 142, r. 164, c. 48.

267. "España en nombre de Bernardino Cuadra Lombera contra los Estados Unidos Mexicanos."

Año: 1932

Estado: Coahuila

AHEEM, CMHMR: MP 142, r. 164, c. 48.

268. "España en nombre de Juan José Garamendia contra los Estados Unidos Mexicanos."

Año: 1932

Estado: Durango

AHEEM, CMHMR: MP 142, r. 164, c. 49.

269. "España en nombre de Garamendia Hermanos contra los Estados Unidos Mexicanos."

Año: 1932

Estado: Durango

AHEEM, CMHMR: MP 142, r. 164, c. 49.

270. "España en nombre de Daniel González, hoy sus herederos, contra los Estados Unidos Mexicanos."

Año: 1932

Estado: San Luis Potosí

AHEEM, CMHMR: MP 142, r. 164, c. 49.

271. "España en nombre de Francisco Romano, sucesión, contra los Estados Unidos Mexicanos."

Año: 1932

Estado: Guerrero

AHEEM, CMHMR: MP 142, r. 164, c. 49.

272. "España en nombre de Ramón González, hoy sus herederos, contra los Estados Unidos Mexicanos."

Añ่o: 1932 
Estado: Guanajuato

AHEEM, CMHMR: MP 142, r. 164, c. 49.

273. "España en nombre de Joaquín

C. Bárcena contra los Estados Unidos Mexicanos."

Año: 1930

Estado: Chihuahua

AHEEM, CMHMR: MP 142, r. 165, c. 50.

274. "España en nombre de Federico González Feito contra los Estados Unidos Mexicanos."

Año: 1932

Estado: Veracruz

AHEEM, CMHMR: MP 142, r. 165, c. 50.

275. "España en nombre de Ramón García López contra los Estados Unidos Mexicanos."

Año: 1932

Estado: Campeche

AHEEM, CMHMR: MP 142, r. 165, c. 50.

276. "España en nombre de Luis García contra los Estados Unidos Mexicanos."

Año: 1931

Estado: Chihuahua

AHEEM, CMHMR: MP 142, r. 165, c. 50.

277. "España en nombre de J. F. González Hermanos contra los Estados Unidos Mexicanos."

Año: 1932

Estado: Veracruz

AHEEM, CMHMR: MP 142, r. 165, c. 50.

278. "España en nombre de Benigno García contra los Estados Unidos Mexicanos."

Año: s.f.

Estado: Jalisco

AHEEM, CMHMR: MP 142, r. 165, c. 51.
279. "España en nombre de Rodríguez

y García contra los Estados Unidos Mexicanos."

Año: 1932

Estado: Coahuila

AHEEM, CMHMR: MP 142, r. 165, c. 51.

280. "España en nombre de Gerónimo

Gil Paredes contra los Estados Unidos Mexicanos."

Año: 1931

Estado: Guanajuato

AHEEM, CMHMR: MP 142, r. 165, c. 51.

281. "España en nombre de Anacleto García contra los Estados Unidos Mexicanos."

Año: 1932

Estado: Puebla

AHEEM, CMHMR: MP 142, r. 165, c. 51.

282. "España en nombre de Lucas Haces Madrid contra los Estados Unidos Mexicanos."

Año: 1931

Estado: Nuevo León

AHEEM, CMHMR: MP 142, r. 165, c. 61.

283. "España en nombre de Bernardo Humara, hoy su testamentaría, contra los Estados Unidos Mexicanos."

Año: 1932

Estado: San Luis Potosí

AHEEM, CMHMR: MP 142, r. 165, c. 51.

284. "España en nombre de Natalio Herrera contra los Estados Unidos Mexicanos."

Año: 1932

Estado: Distrito Federal

AHEEM, CMHMR: MP 142, r. 165, c. 51.

285. "España en nombre de Isidro 
Iglesias Ramos, hoy sus herederos, contra los Estados Unidos Mexicanos." Año: 1932

Estado: Veracruz

AHEEM, CMHMR: MP 142, r. 165, c. 52.

286. "España en nombre de Manuel Sánchez, hoy sus herederos, de contra los Estados Unidos Mexicanos."

Año: 1932

Estado: Tabasco

AHEEM, CMHMR: MP 142, r. 166, c. 52.

287. "España en nombre de Manuel Sánchez, hoy sus herederos, contra los Estados Unidos Mexicanos."

Año: 1932

Estado: Tabasco

AHEEM, CMHMR: MP 142, r. 166, c. 52.

288. "España en nombre de Ángel Irigoyen contra los Estados Unidos Mexicanos."

Año: 1930

Estado: Michoacán

AHEEM, CMHMR: MP 142, r. 166, c. 52.

289. "España en nombre de Alberto Lombrera Camino contra los Estados Unidos Mexicanos."

Año: 1930

Estado: Durango

AHEEM, CMHMR: MP 142, r. 166, c. 52.

290. "España en nombre de Ángel López contra los Estados Unidos Me. xicanos."

Año: 1930

Estado: Coahuila

AHEEM, CMHMR: MP 142, r. 166, c. 53.

291. "España en nombre de José López Gómez contra los Estados Unidos Mexicanos."
Año: 1932

Estado: Tlaxcala

AHEEM, CMHMR: MP 142, r. 166, c. 53.

292. "España en nombre de Manuel Lombrera contra los Estados Unidos Mexicanos."

Año: 1932

Estado: Veracruz

AHEEM, CMHMR: MP 142, r. 166, c. 53.

293. "España en nombre de Manuel Luaces Caballero contra los Estados Unidos Mexicanos."

Año: 1932

Estado: Distrito Federal

AHEEM, CMHMR: MP 142, r. 166, c. 53.

294. "España en nombre de sucesión 'Baldomero Linaje' contra los Estados Unidos Mexicanos."

Año: 1931

Estado: Puebla

AHEEM, CMHMR: MP 142, r. 166, c. 54.

295. "España en nombre de Emilio García Lobato contra los Estados Unidos Mexicanos."

Año: 1932

Estado: San Luis Potosí

AHEEM, CMHMR: MP 142, r. 167, c. 54.

296. "España en nombre de Juan J. Castaños, sucesión, contra los Estados Unidos Mexicanos."

Año: 1932

Estado: Tamaulipas

AHEEM, CMHMR: MP 142, r. 167, c. 55.

297. "España en nombre de Gonzalo Lavín, hoy sus herederos, contra los Estados Unidos Mexicanos."

Año: 1932 
Estado: Coahuila

AHEEM, CMHMR: MP 142, r. 167, c. 55.

298. "España en nombre de Cosme Landeras contra los Estados Unidos Mexicanos."

Año: 1932

Estado: Distrito Federal

AHEEM, CMHMR; MP 142, r. 167, c. 55.

299. "España en nombre de Orosio H. García contra los Estados Unidos Mexicanos."

Año: 1931

Estado: Guanajuato

AHEEM, CMHMR: MP 142, r. 167, c. 55.

300. "España en nombre de Ángel Lan. cerica Cue contra los Estados Unidos Mexicanos."

Año: 1932

Estado: Estado de México

AHEEM, CMHMR: MP 142, r. 167, c. 55.

301. "España en nombre de Aníbal Larrauri contra los Estados Unidos Mexicanos."

Año: 1930

Estado: Guanajuato

AHEEM, CMHMR: MP 142, r. 167, c. 56.

302. "España en nombre de José López

Sáinz contra los Estados Unidos Mexicanos."

Año: 1932

Estado: Veracruz

AHEEM, CMHMR: MP 142, r. 167, c. 56.

303. "España en nombre de Francisco López Pernas contra los Estados Unidos Mexicanos."

Año: 1931

Estado: Chiapas

AHEEM, CMHMR: MP 142, r. 167, c. 56.
304. "España en nombre de Alfonso Llavona contra los Estados Unidos Mexicanos."

Año: 1932

Estado: Veracruz

AHEEM, CMHMR: MP 142, r. 168, c. 57.

305. "España en nombre de Clementina Llano viuda de Gavila contra los Estados Unidos Mexicanos."

Año: 1932

Estado: Jalisco

AHEEM, CMHMR: MP 142, r. 168, c. 57.

306. "España en nombre de Canuto Llarena contra los Estados Unidos Mexicanos."

Año: 1932

Estado: Tamaulipas

AHEEM, CMHMR: MP 142, r. 168, c. 57.

307. "España en nombre de José Jaume Guinart contra los Estados Unidos Mexicanos."

Año: 1932

Estado: Durango

AHEEM, CMHMR: MP 142, r. 168, c. 57.

308. "España en nombre de Eloy Me. taca Fernández contra los Estados Unidos Mexicanos."

Año: 1931

Estado: Estado de México

AHEEM, CMHMR: MP 142, r. 168, c. 58.

309. "España en nombre de Cecilio Mar Cuesta contra los Estados Unidos Mexicanos."

Año: 1932

Estado: Distrito Federal

AHEEM, CMHMR: MP 142, r. 168, c. 58.

310. "España en nombre de Paula Ra- 
mona Mayo viuda de Grande, hoy sus herederos, contra los Estados Unidos Mexicanos."

Año: 1932

Estado: Durango

AHEEM, CMHMR: MP 142, r. 168, c. 58.

311. "España en nombre de Alejandro Martínez, hoy sus herederos, contra los Estados Unidos Mexicanos."

Año: 1932

Estado: Coahuila

AHEEM, CMHMR: MP 142, r. 169, c. 59.

312. "España en nombre de Francisco Mena contra los Estados Unidos Mexicanos."

Año: 1932

Estado: San Luis Potosí

AHEEM, CMHMR: MP 142, r. 169, c. 59.

313. "España en nombre de Antonio Suárez González, sucesión, contra los Estados Unidos Mexicanos."

Año: 1932

Estado: Tabasco

AHEEM, CMHMR: MP 142, r. 169, c. 59.

314. "España en nombre de Isauro Morgadanes, hoy sus herederos, contra los Estados Unidos Mexicanos."

Año: 1932

Estado: Distrito Federal

AHEEM, CMHMR: MP 142, r. 169, c. 59.

315. "España en nombre de Antonio Zorrilla y Luz Manzano de Zorrilla contra los Estados Unidos Mexicanos." Año: 1932

Estado: San Luis Potosí

AHEEM, CMHMR: MP 142, r. 169, c. 59.

316. "España en nombre de Anto- nio, Josefa, Brígida, Luz, María del Carmen, Isabel, Buenaventura, Juan y José María Maqua y Carrizo contra los Estados Unidos Mexicanos."

Año: 1932

Estado: Tlaxcala

AHEEM, CMHMR: MP 142, r. 169, c. 59.

317. "España en nombre de José Novo contra los Estados Unidos Mexicanos." Año: 1932

Estado: Michoacán

AHEEM, CMHMR: MP 142, r. 170, c. 60.

318. "España en nombre de Ramón Maceiras contra los Estados Unidos Mexicanos."

Año: 1932

Estado: Chiapas

AHEEM, CMHMR: MP 142, r. 170, c. 61.

319. "España en nombre de Manuel Martínez, hoy sus herederos, contra los Estados Unidos Mexicanos."

Año: 1932

Estado: Veracruz

AHEEM, CMHMR: MP 142, r. 170, c. 61.

320. "España en nombre de Augusto Madriñán contra los Estados Unidos Mexicanos."

Año: 1932

Estado: Michoacán

AHEEM, CMHMR: MP 142, r. 170, c. 61.

321. "España en nombre de Benito Martínez contra los Estados Unidos Mexicanos."

Año: 1932

Estado: Chihuahua

AHEEM, CMHMR: MP 142, r. 170 , c. 60.

322. "España en nombre de Félix 
Madrazo contra los Estados Unidos Mexicanos."

Año: 1930

Estado: San Luis Potosí

AHEEM, CMHḾR: MP 142, r. 170, c. 62.

323. "España en nombre de Manuel

Melendi contra los Estados Unidos Mexicanos."

Año: 1930

Estado: San Luis Potosí

AHEEM, CMHMR: MP 142, r. 170, c. 62.

324. "España en nombre de Agustín Núñez contra los Estados Unidos Mexicanos."

Año: 1931

Estado: Distrito Federal

AHEEM, CMHMR: MP 142, r. 170, c. 62.

325. "España en nombre de Enrique Niubo Cardona contra los Estados Unidos Mexicanos."

Año: 1932

Estado: Distrito Federal

AHEEM, CMHMR: MP 142, r. 171, c. 63.

326. "España en nombre de Cristino Marquiegui y Andrés Novo contra los Estados Unidos Mexicanos."

Año: 1932

Estado: Guanajuato

AHEEM, CMHMR: MP 142, r. 171, c. 63.

327. "España en nombre de Juan Novales contra los Estados Unidos Mexicanos."

Año: 1931

Estado: Distrito Federal

AHEEM, CMHMR: MP 142, r. 171, c. 63.

328. "España en nombre de Celedonio Niembro contra los Estados Unidos Mexicanos."
Año: 1932

Estado: Morelos

AHEEM, CMHMR: MP 142, r. 171, c. 63.

329. "España en nombre de Ramón Noriega contra los Estados Unidos Mexicanos."

Año: 1931

Estado: Tabasco

AHEEM, CMHMR: MP 142, r. 171, c. 63.

330. "España en nombre de Juan Bautista Ocharán contra los Estados Unidos Mexicanos."

Año: 1930

Estado: Tabasco

AHEEM, CMHMR: MP 142, r. 171, c. 63.

331. "España en nombre de Eugenio Pereda y de la Peña, hoy su representación legal, contra los Estados Unidos Mexicanos."

Año: 1932

Estado: Tlaxcala

AHEEM, CMHMR: MP 142, r. 171, c. 64.

332. "España en nombre de Francisco Porres Peña contra los Estados Unidos Mexicanos."

Año: 1932

Estado: Tamaulipas

AHEEM, CMHMR: MP 142, r. 171, c. 64.

333. "España en nombre de María Bustillo de Del Prado contra los Estados Unidos Mexicanos."

Año: 1932

Estado: Distrito Federal

AHEEM, CMHMR: MP 142, r. 171, c. 64.

334. "España en nombre de Marcial del Prado contra los Estados Unidos Mexicanos." 
Año: 1932

Estado: Guanajuato

AHEEM, CMHMR: MP 142, r. 171, c. 64.

335. "España en nombre de Guadalupe Presno de Martínez Pando contra los Estados Unidos Mexicanos."

Año: 1932

Estado: Tlaxcala

AHEEM, CMHMR: MP 142, r. 172, c. 65.

336. "España en nombre de Natalia Presno y Pérez contra los Estados Unidos Mexicanos."

Año: 1932

Estado: Puebla

AHEEM, CMHMR: MP 142, r. 172, c. 65.

337. "España en nombre de Juan Presno y Pérez contra los Estados Unidos Mexicanos."

Año: 1932

Estado: Puebla

AHEEM, CMHMR: MP 142, r. 172, c. 65.

338. "España en nombre de Marcelino G. de Presno contra los Estados Unidos Mexicanos."

Año: 1932

Estado: Puebla

AHEEM, CMHMR: MP 142, r. 172, c. 65.

339. "España en nombre de Juan Presno y Pérez contra los Estados Unidos Mexicanos."

Año: 1932

Estado: Puebla

AHEEM, CMHMR: MP 142, r. 172, c. 65.

340. "España en nombre de José Pérez y Pérez contra los Estados Unidos Mexicanos."

Año: 1932
Estado: San Luis Potosí

AHEEM, CMHMR: MP 142, r. 172, c. 65.

341. "España en nombre de Francisco Posada, hoy sus herederos, contra los Estados Unidos Mexicanos."

Año: 1932

Estado: Veracruz

AHEEM, CMHMR: MP 142, r. 172, c. 66.

342. "España en nombre de Juan Pando contra los Estados Unidos Mexicanos."

Año: 1932

Estado: Tlaxcala

AHEEM, CMHMR: MP 142, r. 172, c. 66.

343. "España en nombre de Buenaventura Prats contra los Estados Unidos Mexicanos."

Año: 1931

Estado: Durango

AHEEM, CMHMR: MP 142, r. 173, c. 66.

344. "España en nombre de Benigno Fernández P. contra los Estados Unidos Mexicanos."

Año: 1931

Estado: Veracruz

AHEEM, CMHMR: MP 142, r. 173, c. 67.

345. "España en nombre de Sérvulo Higuera contra los Estados Unidos Mexicanos."

Año: 1930

Estado: Guanajuato

AHEEM, CMHMR: MP 142, r. 173, c. 67.

346. "España en nombre de José Pérez Alonso contra los Estados Unidos Mexicanos."

Año: 1931

Estado: Estado de México

AHEEM, CMHMR: MP 142, r. 173, c. 67. 
347. "España en nombre de Francisco Raig contra los Estados Unidos Mexicanos."

Año: 1932

Estado: Chihuahua

AHEEM, CMHMR: MP 142, r. 173, c. 68.

348. "España en nombre de José $S$.

Ramallal contra los Estados Unidos Mexicanos."

Año: 1932

Estado: Distrito Federal

AHEEM, CMHMR: MP 142, r. 173, c. 69.

349. "España en nombre de Prudencio

Rojo de Pedro contra los Estados Unidos Mexicanos."

Año: 1932

Estado: Distrito Federal

AHEEM, CMHMR: MP 142, r. 173, c. 69.

350. "España en nombre de Tomás

Roldán Salvadores contra los Estados Unidos Mexicanos."

Año: 1932

Estado: Estado de México

AHEEM, CMHMR: MP 142, r. 173, c. 69.

351."España en nombre de Juan Ferrer y Cía contra los Estados Unidos Mexicanos."

Año: s.f.

Estado: Tabasco

AHIEEM, CMHMR: MP 142, r. 174, c. 69.

352. "España en nombre de José Ruiz Pardo contra los Estados Unidos Mexicanos."

Año: 1930

Estado: Estado de México

AHEEM, CMHMR: MP 142, r. 174, c. 69.

353. "España en nombre de Mateo
Ruiz Ortiz contra los Estados Unidos Mexicanos."

Año: 1932

Estado: San Luis Potosí

AHEEM, CMHMR: MP 142, r. 174, c. 69.

354. "España en nombre de Remigio Regalado Pérez contra los Estados Unidos Mexicanos."

Año: 1931

Estado: Tamaulipas

AHEEM, CMHMR: MP 142, r. 174, c. 69.

355. "España en nombre de Fidel Ruiz contra los Estados Unidos Mexicanos."

Año: 1932

Estado: San Luis Potosí

AHEEM, CMHMR: MP 142, r. 174, c. 70.

356. "España en nombre de Ulpiano Ruiz Lavín contra los Estados Unidos Mexicanos."

Año: 1930

Estado: Coahuila

AHEEM, CMHMR: MP 142, r. 174, c. 70.

357. "España en nombre de Esteban Roces Llaneza contra los Estados Unidos Mexicanos."

Año: 1932

Estado: Coahuila

AHEEM, CMHMR: MP 142, r. 174, c. 70.

358. "España en nombre de Aurelio Ruiz Ocejo contra los Estados Unidos Mexicanos."

Año: 1930

Estado: Guanajuato

AHEEM, CMHMR: MP 142, r. 174, C. 70.

359. "España en nombre de Felipe y Vicente Renero Cicero contra los Estados Unidos Mexicanos." 
Año: 1932

Estado: Chiapas

AHEEM, CMHMR: MP 142, r. 175, c. 71.

360. "España en nombre de Agustín Rojí contra los Estados Unidos Mexicanos."

Año: 1931

Estado: Puebla

AHEEM, CMHMR: MP 142, r. 175, c. 71.

361. "España en nombre de Ramón Sotres contra los Estados Unidos Mexicanos."

Año: 1932

Estado: Distrito Federal

AHEEM, CMHMR: MP 142, r. 175, c. 71.

362. "España en nombre de José Sordo Mijares contra los Estados Unidos Mexicanos."

Año: 1932

Estado: Distrito Federal

AHEFM, CMHMR: MP 142, r. 175, c. 71.

363. "España en nombre de Juan Salvadores contra los Estados Unidos Mexicanos."

Año: 1931

Estado: Estado de México

AHEFM, CMHMR: MP 142, r. 175, c. 72.

364. "España en nombre de Manuel Stampa contra los Estados Unidos Mexicanos."

Año: 1932

Estado: Distrito Federal

AHEEM, CMHMR: MP 142, r. 175, c. 72.

365. "España en nombre de Ramón Santoveña Inguanzo contra los Estados Unidos Mexicanos."

Año: 1929
Estado: Distrito Federal

AHEEM, CMHMR: MP 142, r. 175, c. 72.

366. "España en nombre de Miguel Solares contra los Estados Unidos Mexicanos."

Año: 1931

Estado: Distrito Federal

AHEEM, CMHMR: MP 142, r. 175, c. 72.

367. "España en nombre de Manuel Salvadores contra los Estados Unidos Mexicanos."

Año: 1932

Estado: Estado de México

AHEEM, CMHMR: MP 142, r. 175, c. 72

368. "España en nombre de Bernardo Santoveña contra los Estados Unidos Mexicanos."

Año: 1932

Estado: Michoacán

AHEEM, CMHMR: MP 142, r. 175, c. 72.

369. "España en nombre de Bartolomé Salva contra los Estados Unidos Mexicanos."

Año: s.f.

Estado: Tabasco

AHEEM, CMHMR: MP 142, r. 175, c. 72.

370. "España en nombre de Ramón Sánchez de la Vega contra los Estados Unidos Mexicanos."

Año: 1930

Estado: San Luis Potosí

AHEEM, CMHMR: MP 142, r. 175, c. 73.

371. "España en nombre de Guadalupe Soberón viuda de Hernández contra los Estados Unidos Mexicanos."

Año: 1932

Estado: San Luis Potosí

AHEEM, CMHMR: MP 142, r. 175, c. 73. 
372. "España en nombre de Pura y Dolores Sarralde contra los Estados Unidos Mexicanos."

Año: 1932

Estado: Veracruz

AHEEM, CMHMR: MP 142, r. 176, c. 73.

373. "España en nombre de Ulpiano Sañudo contra los Estados Unidos Mexicanos."

Año: 1932

Estado: Michoacán

AHEEM, CMHMR: MP 142, r. 176, c. 74.

374. "España en nombre de Federico Sisniega contra los Estados Unidos Mexicanos."

Año: 1932

Estado: Chihuahua

AHEEM, CMHMR: MP 142, r. 176, c. 74.

375. "España en nombre de Sebastián Sáinz Pena contra los Estados Unidos Mexicanos."

Año: 1931

Estado: Guanajuato

AHEEM, CMHMR: MP 142, r. 176, c. 75.

376. "España en nombre de José San Román y Arzuaga contra los Estados Unidos Mexicanos."

Año: 1932

Estado: Nuevo León

AHEEM, CMHMR: MP 142, r. 177, c. 75.

377. "España en nombre de Carmen

San Román de Vázquez contra los Estados Unidos Mexicanos."

Año: 1932

Estado: Guanajuato

AHEEM, CMHMR: MP 142, r. 177, c. 75.

378. "España en nombre de Luis Sáinz contra los Estados Unidos Mexicanos."

Año: 1932

Estado: Estado de México

AHEEM, CMHMR: MP 142, r. 177, c. 76.

379. "España en nombre de Manuel

Salazar Benítez contra los Estados Unidos Mexicanos."

Año: s.f.

Estado: Distrito Federal

AHEEM, CMHMR: MP 142, r. 177, c. 76.

380. "España en nombre de Félix Ubierna contra los Estados Unidos Mexicanos."

Año: s.f.

Estado: Distrito Federal

AHEEM, CMHMR: MP 142, r. 177, c. 76.

381. "España en nombre de Jerónimo Villanueva contra los Estados Unidos Mexicanos."

Año: 1932

Estado: Tabasco

AHEEM, CMHMR: MP 142 , r. 177 , c. 76.

382. "España en nombre de Zavala Hermanos y Cía. contra los Estados Unidos Mexicanos."

Año: 1932

Estado: San Luis Potosí

AHEEM, CMHMR: MP 142, r. 177, c. 76.

383. "España en nombre de Pedro Zabalgoitia, hoy sus herederos, contra los Estados Unidos Mexicanos."

Año: 1932

Estado: Durango

AHEEM, CMHMR: MP 142, r. 177, c. 76.

384. "España en nombre de Ricardo de la Horga contra los Estados Unidos Mexicanos." 
Año: 1931

Estado: Hidalgo

AHEEM, CMHMR: MP 142, r. 177, c. 76.

385. "España en nombre de Manuel Fernández García contra los Estados Unidos Mexicanos."

Año: 1930

Estado: Veracruz

AHENM, CMHMR: MP 142, r. 177, c. 77.

386. "España en nombre de Manuel González Álvarez contra los Estados Unidos Mexicanos."

Año: s.f.

Estado: Tabasco

AHEEM, CMHMR: MP 142, r. 178, c. 77.

387. "España en nombre de Antonio Escondrillas contra los Estados Unidos Mexicanos."

Año: 1932

Estado: Puebla

AHEEM, CMHMR: MP 142, r. 178, c. 77.

388. "España en nombre de Rafael Suárez contra los Estados Unidos Mexicanos."

Año: 1932

Estado: Puebla

AHEEM, CMHMR: MP 142, r. 178, c. 77.

389. "España en nombre de Artenio Muñiz, hoy sus herederos, contra los Estados Unidos Mexicanos."

Año: 1932

Estado: Puebla

AHEEM, CMHMR: MP 142, r. 178, c. 77.

390. "España en nombre de Enrique Lago, hoy sus herederos, contra los Estados Unidos Mexicanos."

Año: 1932
Estado: Puebla

AHEEM, CMHMR: MP 142, r. 178, c. 77.

391. "España en nombre de Francisco Noriega, hoy sus herederos, contra los Estados Unidos Mexicanos."

Año: 1932

Estado: Puebla

AHEEM, CMHMR: MP 142, r. 178, c. 77.

392. "España en nombre de Leonardo Rivero Sotelino, hoy sus herederos, contra los Estados Unidos Mexicanos."

Año: 1932

Estado: Puebla

AHEEM, CMHMR: MP 142, r. 178, c. 77.

393. "España en nombre de José Yarzabal, hoy sus herederos, contra los Estados Unidos Mexicanos."

Año: 1932

Estado: Puebla

AHEEM, CMHMR: MP 142, r. 178, c. 77.

394. "España en nombre de Francisco González, hoy sus herederos, contra los Estados Unidos Mexicanos."

Año: 1932

Estado: Puebla

AHEEM, CMHMR: MP 142, r. 178, c. 77.

395. "España en nombre de Francisco Gómez Solana, sucesión, contra los Estados Unidos Mexicanos."

Año: 1932

Estado: Puebla

AHEEM, CMHMR: MP 142, r. 178, c. 78.

396. "España en nombre de Adolfo González Ubieta contra los Estados Unidos Mexicanos."

Año: 1929

Estado: San Luis Potosí

AHEEM, CMHMR: MP 142, r. 178, c. 78. 
397. "España en nombre de Manuel Diego contra los Estados Unidos Mexicanos."

Año: 1932

Estado: Coahuila

AHEEM, CMHMR: MP 142, r. 178, c. 78.

398. "España en nombre de Alberto Abiega contra los Estados Unidos Mexicanos."

Año: 1932

Estado: Estado de México

AHEEM, CMHMR: MP 142, r. 178, c. 78.

399. "España en nombre de José de Abiega, hoy su sucesión, contra los Estados Unidos Mexicanos."

Año: 1932

Estado: Estado de México

AHEEM, CMHMR: MP 142, r. 178, c. 79.

400. "España en nombre de Julián Ramirez Rasines contra los Estados Unidos Mexicanos."

Año: 1932

Estado: Guanajuato

AHEEM, CMHMR: MP 142, r. 179, c. 79.

401. "España en nombre de María Presas Sobrino contra los Estados Unidos Mexicanos."

Año: 1932

Estado: Estado de México

AHEEM, CMHMR: MP 142, r. 179, c. 79.

402. "España en nombre de Casto Llaca contra los Estados Unidos Mexicanos."

Año: 1930

Estado: Distrito Federal

AHEEM, CMHMR: MP 142, r. 179, c. 79.

403. "España en nombre de Feliciano
Cobián contra los Estados Unidos Mexicanos."

Año: 1932

Estado: Durango

AHEEM, CMHMR: MP 142, r. 179, c. 80.

404. "España en nombre de Donato Blasco Muriel y esposa contra los Estados Unidos Mexicanos."

Año: 1932

Estado: Aguascalientes

AHEEM, CMHMR: MP 142, r. 179, c. 80.

405. "España en nombre de Ignacio Gómez Iturbe contra los Estados Unidos Mexicanos."

Año: 1930

Estado: Estado de México

AHEEM, CMHMR: MP 142, r. 180, c. 81.

406. "España en nombre de José Isusi contra los Estados Unidos Mexicanos." Año: 1932

Estado: Guanajuato

AHEEM, CMHMR: MP 142, r. 180, c. 81.

407. "España en nombre de Tirso Monreal contra los Estados Unidos Mexicanos."

Año: 1930

Estado: Coahuila

AHEEM, CMHMR: MP 142 , r. 180, c. 81.

408. "España en nombre de Teodoro Piñuela Ariño contra los Estados Unidos Mexicanos."

Año: 1932

Estado: Michoacán

AHEEM, CMHMR: MP 142, r. 180, c. 81.

409. "España en nombre de José País contra los Estados Unidos Mexicanos." Año: 1932 
Estado: Veracruz

AHEEM, CMHMR: MP 142, r. 180, c. 82.

410. "España en nombre de Paulino

Ruiz Peña contra los Estados Unidos Mexicanos."

Año: 1932

Estado: Guanajuato

AHEEM, CMHMR: MP 142, r. 180, c. 82.

411. "España en nombre de Sebastián Sobrino contra los Estados Unidos Mexicanos."

Año: 1932

Estado: Distrito Federal

AHEEM, CMHMR: MP 142, r. 180, c. 82.

412. "España en nombre de Apolinar Rodríguez, hoy su sucesión, contra los Estados Unidos Mexicanos."

Año: 1932

Estado: Puebla

AHEEM, CMHMR: MP 142, r. 180, c. 82.

413. "España en nombre de Arturo Martínez, hoy sus herederos, contra los Estados Unidos Mexicanos."

Año: 1932

Estado: Puebla

AHEEM, CMHMR: MP 142, r. 180, c. 82.

414. "España en nombre de Antonio Sustaeta, hoy su sucesión, contra los Estados Unidos Mexicanos."

Año: 1932

Estado: Oaxaca

AHEEM, CMHMR: MP 142, r. 181, c. 82.

415. "España en nombre de Marcelo Enriquez, hoy sus herederos, contra los Estados Unidos Mexicanos."

Año: 1932

Estado: Guerrero

AHEEM, CMHMR: MP 142, r. 181, c. 83.
416. "España en nombre de Rogelio García Lago, hoy su sucesión, contra los Estados Unidos Mexicanos."

Año: 1932

Estado: Puebla

AHEEM, CMHMR: MP 142, r. 181, c. 83.

417. "España en nombre de comunidad de Ángel Díaz Rubían contra los Estados Unidos Mexicanos."

Año: 1932

Estado: Puebla

AHEEM, CMHMR: MP 142, r. 181, c. 83.

418. "España en nombre de Agustín Erquicia y Arzuaga, hoy su sucesión, contra los Estados Unidos Mexicanos." Año: 1932

Estado: Chihuahua

AHEEM, CMHMR: MP 142, r. 181 , c. 84.

419. "España en nombre de José y Antonio Irigoyen, hoy sus herederos Miguel y Fernando Echenique, contra los Estados Unidos Mexicanos."

Año: 1932

Estado: Michoacán

AHEEM, CMHMR: MP 142, r. 182, c. 85.

420. "España en nombre de Avelino Montes Linaje contra los Estados Unidos Mexicanos."

Año: 1932

Estado: Yucatán

AHEEM, CMHMR: MP 142, r. 182, c. 85.

421. "España en nombre de Eduardo Albafull contra los Estados Unidos Mexicanos."

Año: 1930

Estado: Chihuahua

AHEEM, CMHMR: MP 142, r. 182, c. 86. 
422. "España en nombre de Emilio García Marqués contra los Estados Unidos Mexicanos."

Año: 1931

Estado: Chihuahua

AHEEM, CMHMR: MP 142, r. 182, c. 87.

423. "España en nombre de Antonio Quesada, hoy su sucesión, contra los Estados Unidos Mexicanos."

Año: 1932

Estado: Nayarit

AHEEM, CMHMR: MP 142, r. 183 , c. 87.

424. "España en nombre de Leopoldo Ruiz Ocejo, hoy sus herederos, contra los Estados Unidos Mexicanos."

Año: 1932

Estado: Michoacán

AHEEM, CMHMR: MP 142, r. 183, c. 87.

425. "España en nombre de Manuel Sanz Rodríguez contra los Estados Unidos Mexicanos."

Año: 1932

Estado: Michoacán

AHEEM, CMHMR: MP 142, r. 183, c. 87.

426. "España en nombre de Santiago de la Torre contra los Estados Unidos Mexicanos."

Año: 1932

Estado: Michoacán

AHEEM, CMHMR: MP 142 , r. 183 , c. 87

427. "España en nombre de Pedro Cuervo López contra los Estados Unidos Mexicanos."

Año: 1932

Estado: Puebla

AHEEM, CMHMR: MP 142, r. 183, c. 87.

428. "España en nombre de Nicolás
Junco y Cue, hoy sus herederos, contra los Estados Unidos Mexicanos."

Año: 1932

Estado: Veracruz

AHEEM, CMHMR: MP 142, r. 183, c. 88.

429. "España en nombre de Luis González García contra los Estados Unidos Mexicanos."

Año: 1932

Estado: Distrito Federal

AHEEM, CMHMR: MP 142, r. 183, c. 88.

430. "España en nombre de José Antonio Aguirre contra los Estados Unidos Mexicanos."

Año: 1932

Estado: Estado de México

AHEEM, CMHMR: MP 142, r. 183, c. 88.

431. "España en nombre de Ramiro Abraira Muiña contra los Estados Unidos Mexicanos."

Año: 1931

Estado: Distrito Federal

AHEEM, CMHMR: MP 142 , r. 183 , c. 88.

432. "España en nombre de Ángela Conde viuda de Conde contra los Estados Unidos Mexicanos."

Año: 1932

Estado: Puebla

AHEEM, CMHMR: MP 142 , r. 183 , c. 89.

433. "España en nombre de Lucio y Silvano Muniain contra los Estados Unidos Mexicanos."

Año: 1932

Estado: San Luis Potosí

AHEEM, CMHMR: MP 142, r. 184, c. 89.

434. "España en nombre de Mantecón y Pérez contra los Estados Unidos Mexicanos." 
Año: 1932

Estado: Puebla

AHEEM, CMHMR: MP 142, r. 184 , c. 89.

435. "España en nombre de Gumer. sindo Peña e hijo contra los Estados Unidos Mexicanos."

Año: 1932

Estado: Michoacán

AHEEM, CMHMR: MP 142, r. 184, c. 89.

436. "España en nombre de Lorenzo del Peón contra los Estados Unidos Mexicanos."

Año: 1932

Estado: Veracruz

AHEEM, CMHMR: MP 142, r. 184, c. 89.

437. "España en nombre de Casto Santamaría, hoy sus herederos, contra los Estados Unidos Mexicanos."

Año: 1932

Estado: Veracruz

AHEEM, CMHMR: MP 142 , r. 184, c. 89.

438. "España en nombre de Melchor Trueba García contra los Estados Unidos Mexicanos."

Año: 1932

Estado: Tábasco

AHEEM, CMHMR: MP 142, r. 184, c. 90.

439. "España en nombre de Santos Bárcena contra los Estados Unidos Mexicanos."

Año: 1930

Estado: Veracruz

AIIEEM, CMHMR: MP 142, r. 184, c. 90.

440. "España en nombre de Francisco García Canto contra los Estados Unidos Mexicanos."

Año: 1932
Estado: Veracruz

AHEEM, CMHMR: MP 142, r. 185, c. 91.

441. "España en nombre de Carlos Alonso Miyar contra los Estados Unidos Mexicanos."

Año: 1932

Estado: Puebla

AHEEM, CMHMR: MP 142, r. 185, c. 91.

442. "España en nombre de Alejandro Elvira contra los Estados Unidos Mexicanos."

Año: 1932

Estado: Veracruz

AHEEM, CMHMR: MP 142, r. 185, c. 91.

443. "España en nombre de Miguel Fernández contra los Estados Unidos Mexicanos."

Año: 1932

Estado: Veracruz

AHEEM, CMHMR: MP 142, r. 185, c. 91.

444. "España en nombre de Cándido Menéndez contra los Estados Unidos Mexicanos."

Año: 1932

Estado: Puebla

AHEEM, CMHMR: MP 142, r. 185, c. 91.

445. "España en nombre de Constantino Rivero contra los Estados Unidos Mexicanos."

Año: 1930

Estado: Veracruz

AHEEM, CMHMR: MP 142, r. 185, c. 92.

446. "España en nombre de M. Ripoll y Cía. Sucesores S. en C. contra los Estados Unidos Mexicanos."

Año: 1932

Estado: Tabasco

AHEEM, CMHMR: MP 142, r. 185 , c. 92. 
447. "España en nombre de Trinidad Simón viuda de Díaz contra los Estados Unidos Mexicanos."

Año: 1932

Estado: Veracruz

AHEEM, CMHMR: MP 142, r. 186, c. 92.

448. "España en nombre de Ramón García Mayor contra los Estados Unidos Mexicanos."

Año: 1932

Estado: Veracruz

AHEEM, CMHMR: MP 142, r. 185, c. 92.

449. "España en nombre de José Ruisánchez contra los Estados Unidos Mexicanos."

Año: 1932

Estado: Veracruz

AHEEM, CMHMR: MP 142, r. 185, c. 93.

450. "España en nombre de José Gutiérrez Torre contra los Estados Unidos Mexicanos."

Año: s.f.

Estado: Veracruz

AHEEM, CMHMR: MP 142, r. 185, c. 93.

451. "España en nombre de Aurelio Menéndez contra los Estados Unidos Mexicanos."
Año: 1932

Estado: Distrito Federal

AHEEM, CMHMR: MP 142, r. 185, c. 93.

452. "España en nombre de Joaquín Ciávarriz, hoy sus herederos, contra los Estados Unidos Mexicanos."

Año: 1932

Estado: Guanajuato

AHEEM, CMHMR: MP 142, r. 185, c. 93.

453. "España en nombre de Nicomedes Urra, hoy su representante legal, contra los Estados Unidos Mexicanos."

Año: s.f.

Estado: Michoacán

AHEEM, CMHMR: MP 142, r. 185, c. 93.

454. "España en nombre de Cándido Vallina, hoy sus herederos, contra los Estados Unidos Mexicanos."

Año: 1932

Estado: Distrito Federal

AHEEM, CMHMR: MP 142, r. 186, c. 93.

455. "España en nombre de Eduardo de Pascual contra los Estados Unidos Mexicanos."

Año: 1931

Estado: Coahuila

AHEEM, CMHMR: MP 142, r. 186, c. 1. 